\title{
Future Experiments in Relativistic Heavy Ion Collisions
}

\author{
R Bellwied \\ Physics Department, Wayne State University, 666 West Hancock, Detroit, MI 48201, \\ USA \\ E-mail: bellwied@physics.wayne.edu
}

\begin{abstract}
The measurements at RHIC have revealed a new state of matter, which needs to be further characterized in order to better understand its implications for the early evolution of the universe and QCD. I will show that, in the near future, complementary key measurements can be performed at RHIC, LHC, and FAIR. I will focus on results than can be obtained using identified particles, a probe which has been the basis for this conference over the past three decades. The sophisticated detectors, built and planned, for all three accelerator facilities enable us to measure leptons, photons, muons as well as hadrons and resonances of all flavors almost equally well, which makes these experiments unprecedented precision tools for the comprehensive understanding of the physics of the early universe.
\end{abstract}

\section{Introduction: The lessons from RHIC}

Over the past few years the measurements of anisotropic particle flow and jet quenching at RHIC have revealed a deconfined state of matter at high temperature and partonic density, which is characterized best as a near perfect fluid, i.e. a collective state with an extremely low ratio of shear viscosity to entropy. Recently the experiments at RHIC were able to experimentally verify the original conjecture of a state near the quantum limit through several independent measurements of the $\eta / \mathrm{s}$ ratio. Fig.1 shows a summary of calculations based on $\left\langle\mathrm{p}_{T}\right\rangle$-fluctuation, light and heavy quark elliptic flow, and quenching measurements [1, 2, 3, 4]. These calculations are still model dependent, but it is intriguing to recognize that, if the initial conditions assumed in the models are correct, the new state of matter is not well described by either perturbative QCD or any hadronic model.

This led to the definition of the sQGP, a strongly coupled Quark Gluon Plasma, instead of the weak coupling phase we expected from lattice QCD at sufficiently high initial temperature. Since then recent lattice calculations and their comparison to hard thermal loop calculations have revealed that the conditions at RHIC are not sufficient to reach the weakly coupled limit, but that at LHC energies the system likely approaches the perturbative QCD regime [5]. Fig.2a shows, according to Hirano and Gyulassy [6] 


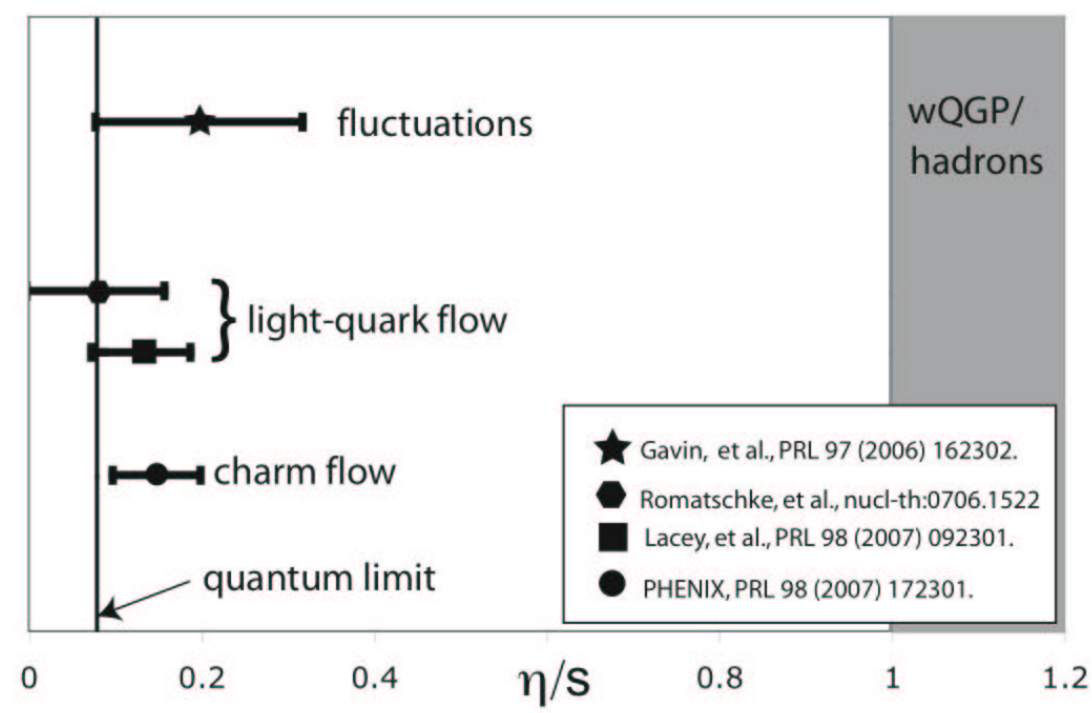

Figure 1. Summary of recent model dependent determinations of the shear viscosity over entropy ratio $(\eta / \mathrm{s})$ based on measurements from STAR and PHENIX.

how the $\eta / \mathrm{s}$ ratio behaves as an order parameter around $\mathrm{T}_{c}$, and Fig.2b speculates on how the difference between LHC and RHIC could be viewed in the QCD phase diagram.

This unexpected behavior of the deconfined matter at RHIC poses several question which drive the physics program of heavy ion collisions to lower and higher collision energies. In my opinion these questions are: 1. What are the degrees of freedom in the partonic liquid ? 2. What are the thermodynamic properties (e.g. equation of state, thermalization mechanism, response to energy deposition) of the sQGP ? 3. What are the details of the QCD phase transition from a strongly coupled partonic system to a hadronic gas (hadronization mechanism) ? 4. Does the strong coupling phase change when the initial conditions change (higher energy, lower $\mathrm{x}$ ) ? 5. What is the order of the phase transition and is there critical behavior in the QCD phase diagram ? 6. Is there any experimental evidence for chiral symmetry restoration ?

The last point was described by Ioffe [7] in a recent article as the remaining unresolved mystery of QCD, and he rightfully pointed out that heavy ion collisions are the only tool to determine the chiral transition experimentally.

Many of these questions, although asked in the narrow frame of our field, have profound implications for the evolution of the universe. Schwarz has summarized the physics of the first second of the universe in a recent paper [8] and concluded that the QCD phase transition might be central not only in understanding the baryonantibaryon asymmetry but also the generation of dark matter in the universe. A similar conclusion was drawn by Kharzeev and Zhvetnitsky recently [9]. Certainly the theory of $\mathrm{CP}$ violation in the strong sector is quite speculative, but in the time evolution of 

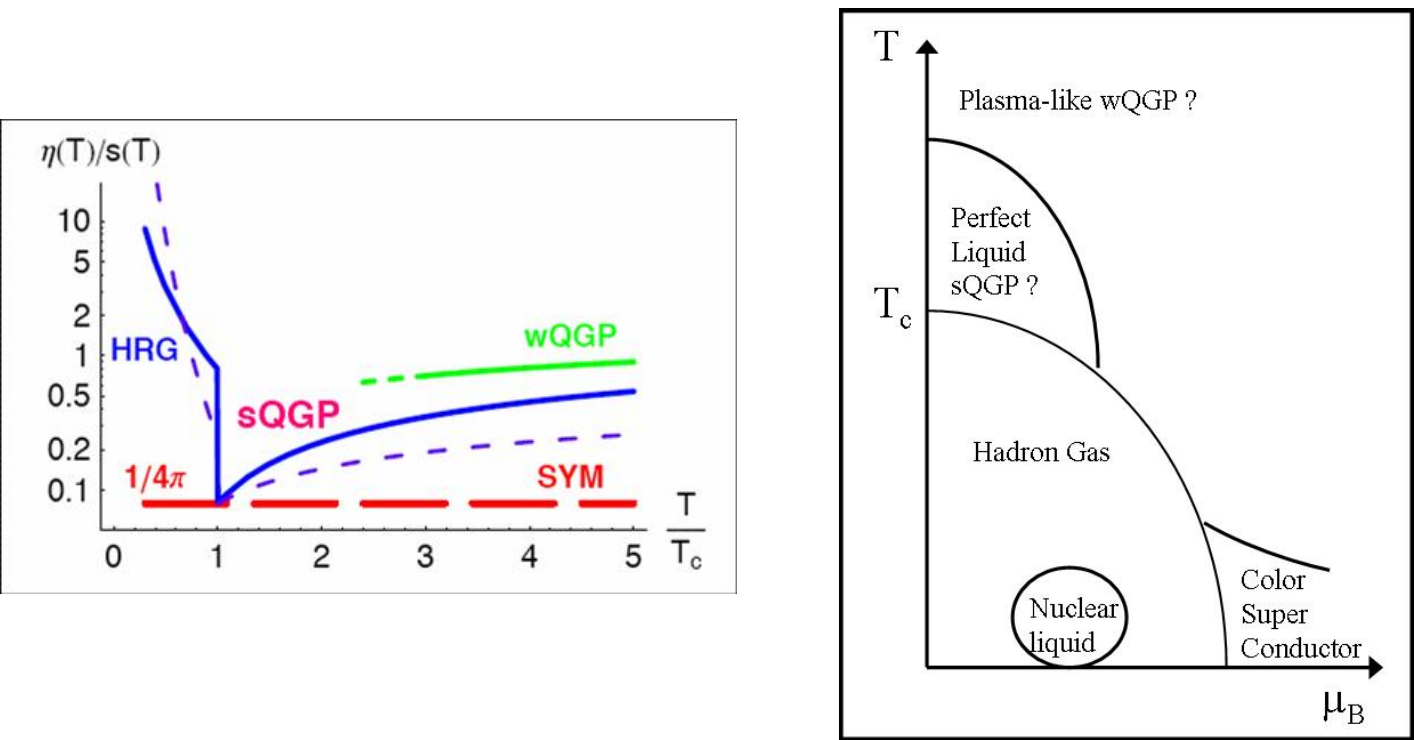

Figure 2. a.) Behavior of $\eta / \mathrm{s}$ as a function of temperature near $\mathrm{T}_{c}$ according to Hirano and Gyulassy [6], b.) New QCD phase diagram based on latest RHIC results.

the early universe the QCD phase transition is situated such that most dark matter candidates have formed but not yet decoupled from the partonic matter [8], and that the baryon-antibaryon asymmetry could be driven by the specifics of the hadronization process at $\mathrm{T}_{c}$.

Federico has summarized the experimental results of the past years in his contribution [10], so here I will only touch on particle identified measurements that are of particular relevance to future experiments.

The particle identified elliptic flow and quenching measurements have revealed a unique scaling in the intermediate transverse momentum region [11, 12]. This so-called constituent quark scaling can be interpreted as evidence for not only deconfinement, but also quark recombination. It is interesting to note that the scaling parameter could be defined as the number of constituent quarks or the number of valence quarks. This shows that the relevant degree of freedom is not well defined. Popular recombination models [13, 14] have taken the approach of using thermalized constituent quarks, with a well defined mass, to describe not only the flow data but also the unexpected high baryon to meson $(\mathrm{B} / \mathrm{M})$ ratio at intermediate $\mathrm{p}_{T}$ in heavy ion collisions. A hump in the proper $\mathrm{p}_{T}$-range in the $\mathrm{B} / \mathrm{M}$ ratio already exists in pp data [15] and can be explained by a di-quark suppression factor for baryon formation, but this mechanism does not allow the baryon yield to exceed the meson yield, whereas quark coalescence explicitly enhances the baryon yield over the meson yield in the intermediate $\mathrm{p}_{T}$-range. This 

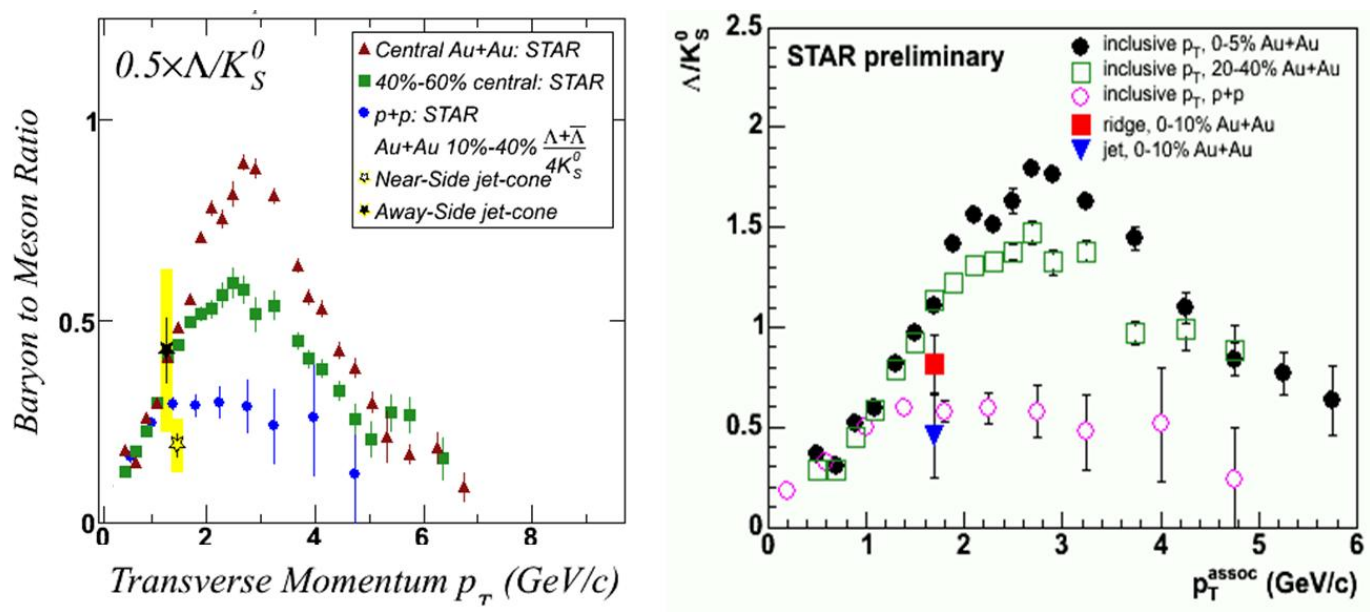

Figure 3. STAR measurements of the inclusive $\Lambda / K_{s}^{0}$ ratio as a function of centrality and transverse momentum, a.) compared to ratios measured in the same-side and away-side jet region from triggered two-particle correlations [16, b.) compared to ratios measured in the same-side jet and the long-range correlation ridge region from triggered two-particle correlations [17].

difference between pp and AA data very much suggests a melting of any multi-quark structure which might have existed in pp in favor of a system of deconfined, possibly thermal, quarks in AA collisions.

Extensions to the simple inclusive $\mathrm{B} / \mathrm{M}$ ratio measurements in heavy ion collisions have been recently performed by the STAR collaboration and were shown at this meeting. Here $\mathrm{B} / \mathrm{M}$ ratios were determined in structures which appeared in high momentum two-particle correlation measurements. Fig.3a shows a comparison of B/M ratios in same-side and away-side jet cones triggered by a high momentum charged particle [16], Fig.3b shows a comparison of $\mathrm{B} / \mathrm{M}$ ratios in the same side jet cone and the same-side long-range correlation ridge as measured by STAR [17]. In both cases it seems that inside the unquenched jet the $\mathrm{B} / \mathrm{M}$ ratio is consistent with expectations from fragmentation models, whereas the in-medium response to the traversing jets, either in the same side ridge or the away side cone, leads to a ratio that is better described by the recombination scenario.

The recent measurement of the nuclear suppression factor and the elliptic flow for D-mesons, based on electrons from the semi-leptonic decay of the heavy mesons [18, 19, 20], allows us to determine the applicability of partonic recombination for heavy quarks, and early results seem to indicate that both, the $\mathrm{R}_{A A}$ and the v2 measurements, can only be explained if one assumes identical $\mathrm{p}_{T}$-dependencies for the flow and the quenching of light and heavy quarks as is shown in Fig.4a for $\mathrm{R}_{A A}$ [21] and in Fig.4b for v2 [11]. In other words, there is a remarkable lack of quark mass dependence in the 

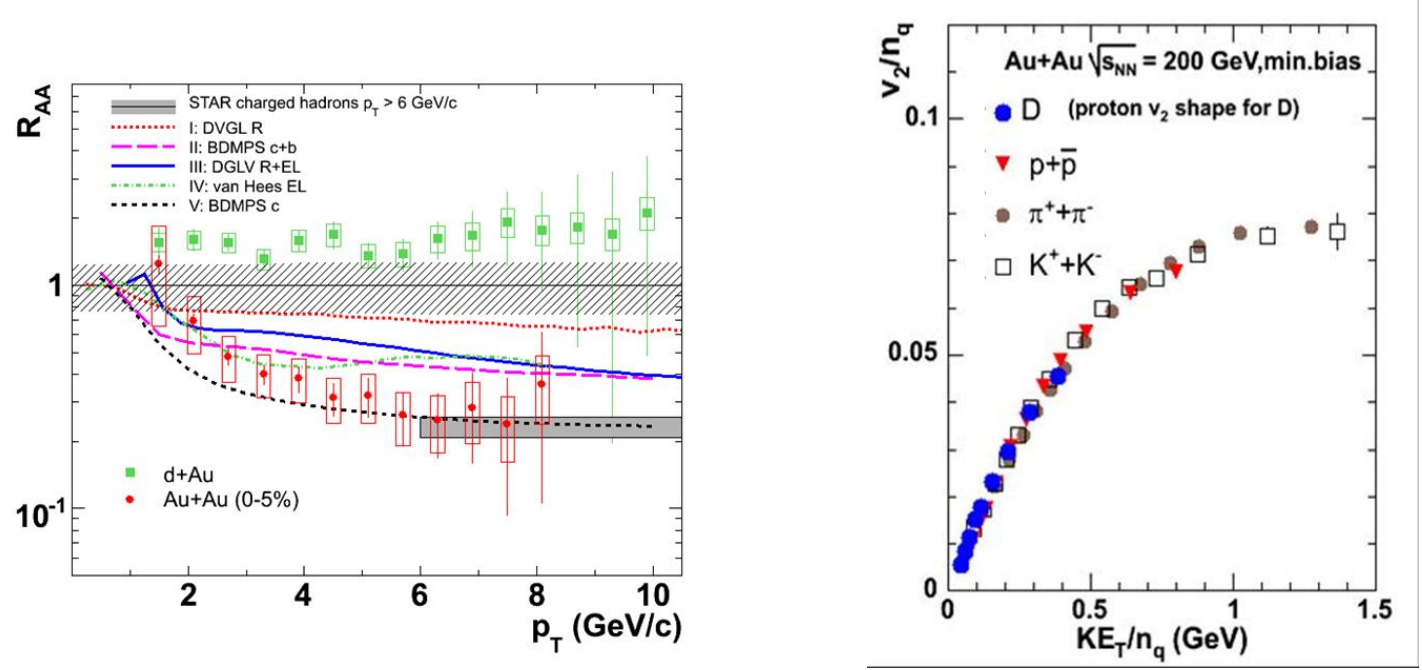

Figure 4. a.) Nuclear suppression factor for non-photonic single electron spectra in semi-central $\mathrm{Au}+\mathrm{Au}$ collisions at RHIC compared to the $\mathrm{R}_{A A}$ for charged hadrons (i.e. light quark suppression) and various models [21. b.) Elliptic flow (non-scaled and $\mathrm{n}_{q}$ scaled) for non-photonic single electron spectra in semi-central $\mathrm{Au}+\mathrm{Au}$ collisions at RHIC compared to light quark hadrons [11.

scaling parameter. At some high momentum the mass of the bare or constituent heavy quark might be negligible, but this should not be the case for the intermediate momenta measured here. Many models, as shown in Fig.4a, have been proposed to address these measurements. The most successful of these models try to give the heavy quark a special status, by postulating either the survival of heavy quark resonant states above $\mathrm{T}_{c}[22,23]$ or the reduced formation time of heavy quark hadrons from the partonic phase [24].

The near identical $\mathrm{p}_{T}$-dependence of the $\mathrm{v} 2$ and the quark energy loss of light and heavy quarks is very striking, though, and might require a much more fundamental explanation. A detailed measurement of reconstructed D-mesons and B-mesons is sorely needed to remove the ambiguities in the semi-leptonic measurements, and future measurements of high momentum heavy flavor mesons and baryons should answer the question whether the liquid phase above the critical temperature requires indeed a special degree of freedom to describe all features of hadronization from a dense deconfined medium. Both STAR and PHENIX have proposed extensive upgrades to their vertexing and tracking capabilities in order to be able to directly reconstruct the displaced vertices of the hadronic decay channels of heavy quark hadrons [25, 26]. 


\section{The FAIR/RHIC low energy program: Is it critical ?}

Besides the very detailed and strong evidence for deconfinement at RHIC energies, the data reveal a surprising lack of evidence for chiral symmetry restoration. Vector meson and resonance measurements have been performed to new levels of precision at RHIC, in particular in the sector of heavy hadronic resonances, but the measurements are mostly used to determine the lifetime of the produced partonic and hadronic systems through detailed mapping of re-interaction probabilities [27], because the properties of the resonances, such as mass, width, and branching ratios are generally in very good agreement with the particle data group references. There are small variations in mass and width for certain resonances as a function of their momentum, but they have been measured consistently in pp, dA, and AA collisions [28, 29] and therefore should not be attributed to chiral symmetry restoration. Recently PHENIX has shown results that might indicate behavior similar to the NA45 and NA60 low mass di-lepton measurements [30], but whether this is evidence for medium modification of vector mesons remains to be seen. It seems that either our measurements are not sensitive to chiral symmetry restoration or that the chiral transition might indeed decouple from deconfinement, which is in disagreement with lattice QCD calculations. A very high luminosity program at FAIR should enable more detailed measurements of medium modification, in particular for chiral partners in the heavy quark sector. Not only will the lifetime of the dense hadronic phase be considerably longer than at RHIC, but, although open charm production is at threshold at FAIR energies, the yield of open charm obtained in a 25 week run at CBM is about an order magnitude larger than the yield STAR obtains over the same period of time [31. Detailed measurements, not only of chiral symmetry restoration, but also particle identified elliptic flow, radial flow and jet quenching might therefore be possible albeit at a slightly lower $\mathrm{p}_{T}$-range. The key purpose of these measurements should be to map out the disappearance of the main sQGP signatures, such as quark scaling, hydro scaling, and high $\mathrm{p}_{T}$ suppression in order to determine the exact phase transition point in the QCD phase diagram. These results will complement the thrust of the CBM program and also the low-energy RHIC program, which focusses on the search for a critical point. In that context, STAR is upgrading its detector to include a full azimuth TOF device to allow the measurements of particle identified yield ratios on an event by event basis. Besides addressing the remarkable structure in the integrated $\mathrm{K}^{+} / \pi^{+}$ratio as function of collision energy, as measured by NA49 [32], the event-by-event $\mathrm{K} / \pi$ ratio will together with $\left\langle\mathrm{p}_{T}\right\rangle$, charge, and baryon number measurements be sensitive to critical dynamic fluctuations.

\section{The LHC program: Vaporizing the Quark Soup}

If the strong coupling strength reaches the weak limit at around $3 \mathrm{~T}_{c}$, then it is very likely that at LHC energies we will indeed reach the plasma, rather than liquid, phase which was originally anticipated for RHIC energies. This phase will only exist for a few 
$\mathrm{fm} / \mathrm{c}$ and then has to de-excite through the strong coupling phase to the hadronization surface, but the question arises whether the weak coupling during the early times might lead to any measurable features. It is likely that the hadronization mechanism is not affected, but collective phenomena which are supposed to develop early, such as elliptic flow, might be reduced by a weak coupling phase. One can also speculate that the system might be more dilute when it enters the strong coupling regime, and therefore exhibits less of a collectivity.

On the other hand, the partonic system is expected to live longer, and estimates by Eskola et al. [33] show that the applicability of hydrodynamics might extend to higher $\mathrm{p}_{T}$ which means that the thermal bulk will begin to populate the intermediate $\mathrm{p}_{T}$ range. At the same time it is likely that the quark coalescence mechanism will push out to higher $\mathrm{p}_{T}$ simply because the thermal partons will carry more energy at LHC energies [34. Finally the increase in jet cross section will lead to enhanced particle production in the intermediate $\mathrm{p}_{T}$ range through jet quenching [35]. In that context, hybrid models which allow the recombination of thermal partons with hard fragmentation partons claim to predict the particle spectrum at the LHC over a wide momentum range (2-20 $\mathrm{GeV} / \mathrm{c}$ ) [36]. Clearly, although the intermediate momentum range is populated much stronger at the LHC than at RHIC, it will be challenging to disentangle all these different contributions to hadron production. On the other hand the large statistics will allow us to study the transition from bulk matter production to non-thermal contributions at higher momentum in a systematic way at the LHC. In particular ALICE is well suited for hadronization studies through its superior particle identification capabilities.

Certain predictions made at this conference have revealed a great sensitivity to the properties of the plasma at the LHC. I will only list a few that I think are of special relevance to the theme of this conference.

The question of equilibrium vs. non-equilibrium at the time of hadronization has still not been settled through the RHIC measurements, but at the LHC the possible oversaturation of strangeness in a non-equilibrium approach leads to a novel effect as shown by Rafelski [37], namely the binding of charm by strange quarks into rare heavy states, such as the $\mathrm{D}_{s}$ and the $\Xi_{c}$

In the heavy sector the ratio of charm quenching over bottom quenching is expected to approach unity at sufficiently high transverse momentum $\left(\mathrm{p}_{T}>50 \mathrm{GeV} / \mathrm{c}\right)$ if $\mathrm{pQCD}$ is correct, whereas a AdS/CFT based calculation shows no sensitivity to the change in collision energy and predicts a constant quenching ratio for $\mathrm{c} / \mathrm{b}$ independent of the particle momenta as shown by Horowitz [38]. A similar cross-check is given by comparing muons from $\mathrm{W}$ decays to muons from $\mathrm{B}$-meson decays. The muons from $\mathrm{W}$ decays are effectively medium blind, whereas the B-meson muons should exhibit the effect of jet quenching. Dainese [39] showed that the crossing point for $\mathrm{W} / \mathrm{B}$ muons in transverse momentum will be sensitive to the level of partonic energy loss for B-mesons in medium.

The measurement of all onium states, and in particular separate measurements of all $\mathrm{Y}$ states will enable a comprehensive look at color screening in the medium. The $\mathrm{J} / \psi$ measurement holds additional information, because the abundant production of 
c-cbar pairs at the LHC increases the probability of partonic recombination of $\mathrm{J} / \psi$ to offset the screening effect. Therefore recombination models have the $\mathrm{J} / \psi$ yield actually rise from RHIC to the LHC [40]. It was interesting to note at this conference, though, that hadronic recombination models, which favor the coalescence of charm bound in D-mesons during the co-moving hadronic phase, yield the same $\mathrm{J} / \psi$ enhancement than the partonic models [41, 42].

Finally, the measurement of resonances in jets as proposed by Markert [43] might hold great promise at a more differential probe of chiral symmetry restoration in the medium. The idea here is based on the possibility that heavy high momentum resonances are formed earlier in the mixed phase than the bulk pions. In this case the resonances might undergo medium modifications in the partonic medium but carry too high a momentum to re-interact hadronically after bulk hadronization. It is intriguing to recognize that the reference measurement of hadronically interacting resonances could be made in the same event, simply by comparing resonances correlated to the jet axis with off axis, or uncorrelated, resonances. STAR will attempt such a measurement but only the increased yield and momentum reach, as well as the longer lifetime of the partonic phase at the LHC is likely to enable us to achieve the necessary conditions.

\section{Future detector upgrades: Are they necessary?}

Possible requirements for new detector components are based on specific shortcomings of the existing detectors for certain key measurements. A comparison of heavy ion detectors to elementary particle detectors reveal some of these features. For example, it might be desirable in a heavy ion experiment to have near hermetic coverage, i.e. detection capabilities should extend to the highest accessible pseudo-rapidity, in particular for decay $\gamma$ and the away-side component of high momentum triggered $\gamma$-jet events. It would also be interesting to have high precision vertexing and tracking out to very high momentum without having to build even larger gas based TPC's. This can be achieved through a solid-state tracker in a large magnetic field. One could further extend the granularity of electromagnetic calorimetry for photon (direct, decay, fragmentation and thermal photons) measurements, which drives the necessity for a crystal type calorimeter and hadronic calorimetry to distinguish the different neutral particle energy contributions. A key benchmark for any final calorimeter design should be that all $\Upsilon$ states and the $\chi_{c}$ can be reconstructed in the heavy-ion environment and that the away-side spectrum of $\gamma$-jets can be reliably measured out to a high $\gamma$ - $\mathrm{p}_{T}$. Finally a new device should include a very high momentum particle identification component to extend the critical flavor dependent measurements out to high momentum. Reliable identified singles spectra should be measured to about $25 \mathrm{GeV} / \mathrm{c}$ in order to be able to distinguish all the different contributions to the spectrum.

These are simple hardware extensions based on the existing limitations of the big three heavy ion detectors (STAR,PHENIX, ALICE), but they also can be easily linked to interesting new measurements required to fully characterize the partonic phase at 
RHIC and the LHC. It is for the next generation of heavy ion physicists to decide whether all these improvements can be realized within a reasonable budget or whether targeted upgrades to existing devices will be sufficient.

\section{Conclusions: The Future is bright}

The future of relativistic heavy ion physics is very bright. The discovery of the sQGP at RHIC has shown that a new phase of matter has existed above the critical temperature during the early evolution of the universe. It is encumbant upon us to characterize this phase in great detail and to explore the likely far-reaching implications for the evolution of matter during the first second of the universe. Both the low energy program at RHIC/FAIR as well as the final frontier program at the LHC are absolutely crucial for understanding this new phase of matter. In addition it might turn out that the RHIC energy constitutes a sweet spot for studying the process of hadronization from strongly coupled partonic matter to hadrons. Therefore all three programs will have a very strong future ahead of them.

\section{References}

[1] S. Gavin and M. Abdel-Aziz, Phys. Rev. Lett. 97, 162302 (2006)

[2] P. Romatschke and U. Romatschke, arXiv:0706.1522

[3] R. Lacey et al., Phys. Rev. Lett. 98, 092301 (2007)

[4] A. Adare et al. (PHENIX coll.), Phys. Rev. Lett. 98, 172301 (2007)

[5] J.P. Blaizot et al., hep-ph/0611393

[6] T. Hirano and M. Gyulassy, Nucl.Phys. A769, 71 (2006)

[7] B.L. Ioffe, hep-ph/0601250

[8] D. Schwarz, Annalen Phys. 12, 220 (2003)

[9] D. Kharzeev and A.Zhtinitsky, arXiv:0706.1026

[10] F. Antinori, contribution to this conference

[11] see e.g. A.Taranenko for PHENIX collaboration, nucl-ex/0703025

[12] S. Salur for STAR coll., nucl-ex/0606006

[13] R.J. Fries et al. Phys. Rev. Lett. 90, 202303 (2003)

[14] V. Greco et al., Phys. Rev. Lett. 90, 202302 (2003)

[15] B. Abelev et al. (STAR coll.), Phys. Rev. C75, 064901 (2007)

[16] J. Zuo, contribution to this conference and arXiv:0705.3286

[17] J. Bielcikova, arXiv:0707.3100

[18] S.S. Adler et al. (PHENIX coll.), Phys.Rev.Lett. 94, 082301 (2005)

[19] J.Bielcik for STAR collaboration, Nucl.Phys. A774, 697 (2006)

[20] S.S. Adler et al., PHENIX collaboration, Phys.Rev. C72, 024901 (2005)

[21] B. Abelev et al., STAR coll., Phys.Rev.Lett. 98, 192301 (2007)

[22] R. Rapp and H. Van Hees, J.Phys. G32, S351 (2006)

[23] H. van Hees et al., Phys. Rev. C73, 034913 (2006)

[24] A. Adil and I. Vitev, Phys.Lett. B649, 139 (2007)

[25] K. Schweda for STAR coll., Nucl.Phys. A774, 907 (2006)

[26] R. Noucier for PHENIX coll., nucl-ex/0703041

[27] B. Abelev et al. (STAR coll.), Phys. Rev. Lett. 97, 132301 (2006)

[28] J. Adams et al. (STAR coll.), Phys. Rev. Lett. 92, 092301 (2004)

[29] P. Fachini, contribution to this conference 
[30] S. Afanasiev et al. (PHENIX coll.), arXiv:0706.3034

[31] P. Senger, contribution at BORMIO 2007

[32] C. Blume, nucl-ex/0610049

[33] K.J. Eskola et al., Nucl.Phys. A774, 805 (2006)

[34] R.J. Fries and B. Mueller, EPJ C34, S279 (2004)

[35] N. Borghini and U. Wiedemann, Nucl.Phys. A774, 549 (2006)

[36] R.C. Hwa and C.B. Yang, Phys.Rev.Lett. 97, 042301 (2006)

[37] J. Rafelski, contribution to this conference and hep-ph/0607203

[38] W.A. Horowitz, contribution to this conference and arXiv:0706.2336

[39] A. Dainese, contribution to this conference

[40] A. Andronic et al., nucl-th/0701079

[41] L. Bravina, contribution to this conference

[42] O. Linnyk, contribution to this conference

[43] C. Markert, arXiv:0706.0724 